\title{
On a class of Humbert-Hermite polynomials
}

\author{
M. A. Pathan ${ }^{1}$ and Waseem A. Khan ${ }^{2}$ \\ ${ }^{1}$ Centre for Mathematical and Statistical Sciences (CMSS), Peechi P.O., Thrissur, \\ Kerala-680653, India \\ ${ }^{2}$ Department of Mathematics, Faculty of Science, Integral University, \\ Lucknow-226026, India \\ E-mail: mapathan@gmail.com, waseem08_khan@rediffmail.com
}

\begin{abstract}
A unified presentation of a class of Humbert's polynomials in two variables which generalizes the well known class of Gegenbauer, Humbert, Legendre, Chebycheff, Pincherle, Horadam, Kinnsy, Horadam-Pethe, Djordjević , Gould, Milovanović and Djordjević, Pathan and Khan polynomials and many not so called 'named' polynomials has inspired the present paper and the authors define here generalized Humbert-Hermite polynomials of two variables. Several expansions of HumbertHermite polynomials, Hermite-Gegenbaurer (or ultraspherical) polynomials and HermiteChebyshev polynomials are proved.
\end{abstract}

Keywords: Hermite polynomials, Humbert polynomials, Gegenbauer polynomials, Chebyshev polynomials, Pathan-Khan polynomials, hypergeometric function.

2010 Mathematics Subject Classification: 33C45, 33C55, 33C99.

\section{Introduction}

The 2-variable Kampé de Fériet generalization of the Hermite polynomials [3] and [7] defined as

$$
H_{n}(x, y)=n ! \sum_{r=0}^{\left[\frac{n}{2}\right]} \frac{y^{r} x^{n-2 r}}{r !(n-2 r) !} .
$$

These polynomials are usually defined by the generating function

$$
e^{x t+y t^{2}}=\sum_{n=0}^{\infty} H_{n}(x, y) \frac{t^{n}}{n !},
$$

and reduce to the ordinary Hermite polynomials $H_{n}(x)$ (see [1]) when $y=-1$ and $x$ is replaced by $2 x$.

Next, we recall the definition of $\mathrm{N}$-variable generalized Hermite polynomials $H_{n}\left(\{x\}_{1}^{N}\right)$ defined by Dattoli et al. [6, p.602]:

$$
\exp \sum_{s=1}^{N} x_{s} t^{s}=\sum_{n=0}^{\infty} H_{n}\left(\{x\}_{1}^{N}\right) \frac{t^{n}}{n !},
$$

where $\{x\}_{1}^{N}=x_{1}, x_{2}, \ldots, x_{N}$.

Generalized Hermite polynomials $H_{n}\left(\{x\}_{1}^{N}\right)$ for $N=3$ also belong to those of Bell type as shown in $\left[8\right.$, p.403(26)]. The Gould-Hooper polynomials $g_{n}^{m}(x, y)$ (see [5] and [10]) is a special case of (1.3). The notation $H_{n}^{m}(x, y)$ or $g_{n}^{m}(x, y)$ was given by Dattoli et al. [5]. These are specified by

$$
e^{x t+y t^{m}}=\sum_{n=0}^{\infty} H_{n}^{m}(x, y) \frac{t^{n}}{n !}
$$


Another generalization of Hermite polynomials which we wish to consider in this paper is given by $H_{n, m, \nu}(x, y)$ in the form of the generating function (see [16])

$$
e^{\nu(x+y) t-(x y+1) t^{m}}=\sum_{n=0}^{\infty} H_{n, m, \nu}(x, y) \frac{t^{n}}{n !},
$$

which reduces to the ordinary Hermite polynomials $H_{n}(x)$ when $\nu=2, x=0$ or $\nu=2, y=0$.

We draw attention to familiar generating relations given by

$$
\left(1-2 x t+t^{2}\right)^{-\frac{1}{2}}=\sum_{n=0}^{\infty} P_{n}(x) t^{n}
$$

where $P_{n}(x)$ is Legendre's polynomial of first kind.

$$
\left(1-2 x t+t^{2}\right)^{-1}=\sum_{n=0}^{\infty} U_{n}(x) t^{n}
$$

where $U_{n}(x)$ is Chebychev polynomial of second kind.

$$
\left(1-2 x t+t^{2}\right)^{-\nu}=\sum_{n=0}^{\infty} C_{n}^{\nu}(x) t^{n}
$$

where $C_{n}^{\nu}(x)$ is Gegenbauer's polynomial.

$$
\begin{gathered}
\left(1-m x t+t^{m}\right)^{-\nu}=\sum_{n=0}^{\infty} h_{n, m}^{\nu}(x) t^{n}, \\
h_{n, m}^{\nu}(x)=\sum_{k=0}^{\left[\frac{n}{m}\right]} \frac{(-1)^{k}(\nu)_{n+(1-m) k}(m x)^{n-m k}}{k !(n-m k) !}
\end{gathered}
$$

where $h_{n, m}^{\nu}(x)$ is Humbert polynomial and $m$ is a positive integer. The Pochammer symbol $(a)_{n}$ is defined by

$$
(a)_{n}=\frac{\Gamma(a+n)}{\Gamma(a)}=\left[\begin{array}{ll}
1 & \text { if } n=0 \\
a(a+1)(a+2) \cdots(a+n-1) & \text { if } n=1,2,3 \cdots
\end{array}\right.
$$

In 1965, Gould [11] gave the following generating relation

$$
\left(c-m x t+y t^{m}\right)^{p}=\sum_{n=0}^{\infty} P_{n}(m, x, y, p, c) t^{n},
$$

where $m$ is a positive integer and other parameters are unrestricted in general. $P_{n}(m, x, y, p, c)$ is defined explicitly by [11, p.699]:

$$
P_{n}(m, x, y, p, c)=\sum_{k=0}^{\left[\frac{n}{m}\right]}\left(\begin{array}{l}
p \\
k
\end{array}\right)\left(\begin{array}{l}
p-k \\
n-m k
\end{array}\right) c^{p-n+(m-1) k} y^{k}(-m x)^{n-m k}
$$

In 1989, Sinha [19] gave the following generating relation

$$
\left[1-2 x t+t^{2}(2 x-1)\right]^{-\nu}=\sum_{n=0}^{\infty} S_{n}^{\nu}(x) t^{n}
$$

where

$$
S_{n}^{\nu}(x)=\sum_{k=0}^{\left[\frac{n}{2}\right]} \frac{(-1)^{k}(\nu)_{n-k}(2 x)^{n-2 k}(2 x-1)^{k}}{k !(n-2 k) !}
$$


$S_{n}^{\nu}(x)$ is the generalization of Shrestha polynomial $S_{n}(x)$ ( see [16]).

In 1991, Milovanović and Djordjević [14](see also [15])gave the following generating relation

$$
\left(1-2 x t+t^{m}\right)^{-\lambda}=\sum_{n=0}^{\infty} p_{n, m}^{\lambda}(x) t^{n}
$$

where $m \in \mathbb{N}$ and $\lambda>-\frac{1}{2}$ and

$$
p_{n, m}^{\lambda}(x)=\sum_{k=0}^{\left[\frac{n}{m}\right]} \frac{(-1)^{k}(\lambda)_{n-(m-1)_{k}}(2 x)^{n-m k}}{k !(n-m k) !} .
$$

It is to be noted that the polynomials represented by $p_{n, 1}^{\lambda}(x), p_{n, 2}^{\lambda}(x)$ and $p_{n, 3}^{\lambda}(x)$ are known as Horadam polynomials [12], Gegenbauer polynomials and Horadam-Pethe polynomials [13], respectively.

Many interesting generalizations to these polynomials appeared in the literature. In particular in 1997, Pathan and Khan [16,p.54] generalized these polynomials and gave the following generating relation

$$
\begin{aligned}
{\left[c-a x t+b t^{m}(2 x-1)^{d}\right]^{-\nu} } & =\sum_{n=0}^{\infty} p_{n, m, a, b, c, d}^{\nu}(x) t^{n} \\
& =\sum_{n=0}^{\infty} \Theta_{n}(x) t^{n},
\end{aligned}
$$

where

$$
\Theta_{n}(x)=\sum_{k=0}^{\left[\frac{n}{m}\right]} \frac{(-1)^{k} c^{-\nu-n+(m-1) k}(\nu)_{n+(1-m) k}(a x)^{n-m k}\left[b(2 x-1)^{d}\right]^{k}}{k !(n-m k) !} .
$$
in the form

Djordjević [9] provided a generalization of various polynomials of two variables

$$
\left[1-2(x+y) t+t^{m}(2 x y+1)\right]^{-\alpha}=\sum_{n=0}^{\infty} G_{n}^{\alpha, m}(x, y) t^{n}
$$

where

$$
G_{n}^{\alpha, m}(x, y)=\sum_{k=0}^{\left[\frac{n}{m}\right]} \frac{(-1)^{k}(\alpha)_{n-(m-1) k}(2 x+2 y)^{n-m k}(2 x y+1)^{k}}{k !(n-m k) !} .
$$

Note that $G_{n}^{1, m}(x, y)=C_{n}^{m}(x, y)$ and $G_{n}^{1 / 2, m}(x, y)=P_{n}^{m}(x, y)$ where $C_{n}^{m}(x, y)$ and $U_{n}^{m}(x, y)$ are Chebyshev and Legendre polynomials of two variables, respectively.

For $m=2, G_{n}^{\alpha, m}(x, y)$ reduces to a polynomial studied by Dave [4]. For $m=2$ and $y=0, G_{n}^{\alpha, m}(x, y)$ reduces to Gegenbauer polynomial and for $m=3$ and $y=0$, $G_{n}^{\alpha, m}(x, y)$ are Horadam-Pethe polynomials [13]. Further, for $y=0, G_{n}^{\alpha, m}(x, y)$ reduces to a polynomial $p_{n, m}^{\alpha}(x)$ studied by Milovanović and Djordjević ([14]and [15]).

A generalization and unification of various polynomials mentioned above is provided by the definition of generalized Humbert polynomials in two variables given 
recently by Pathan and Khan [17] which has the generating function

$$
\left[a-(b x+c y) t+d t^{m}(e x y-1)^{g}\right]^{-h}=\sum_{n=0}^{\infty} Q_{n, m, g, h}^{a, b, c, d, e}(x, y) t^{n}=\sum_{n=0}^{\infty} Q_{n}(x, y) t^{n}
$$

where $m \in \mathbb{N}, h>0$ and the other parameters are unrestricted in general.

In (1.20), if we put $a=1, b=c=2, d=-1, e=-2$ and $g=1$, then we get a generating relation (1.18) studied by Djordjević [9]. For $y=1, e=2$ and $c=0$, we get a generating relation (1.16) studied by Pathan and Khan [16]. For $a=1, b=2$, $c=0, d=1$ and $g=0$, we get a generating relation (1.14) studied by Milovanović Djordjević [15]. For $a=1, b=2, m=2, y=1, e=2$ and $g=1$, we get a polynomial defined by Sinha [19] and for $c=0, g=0, d=y$ and $h=-p$, we get a generating relation (1.4) given by Gould [11]. Some more interesting special cases which are recorded by G.B. Djordjević and G.V. Milovanović in [10] can be established similarly.

\section{On a class of Humbert-Hermite polynomials}

A generalization and unification of various polynomials mentioned above is provided by the definition of generalized Humbert-Hermite polynomials ${ }_{H} G_{n}^{\nu, \alpha, m}(x, y)$ in two variables which has the generating function

$$
\left[1-2(x+y) t+t^{m}(2 x y+1)\right]^{-\nu} e^{\alpha(x+y) t-(x y+1) t^{m}}=\sum_{n=0}^{\infty}{ }_{H} G_{n}^{\nu, \alpha, m}(x, y) t^{n}
$$

where $m \in \mathbb{N}, \alpha, \nu>0$ and the other parameters are unrestricted in general. This is interesting since, as will be shown, the polynomials ${ }_{H} G_{n}^{\nu, \alpha, m}(x, y)$ contain a number of known polynomials (see [4], [9], [10], [11], [12], [13], [14], [16], [17] and [18]).

Using the definitions of $H_{n, m, \nu}(x, y)$ and $G_{n}^{\alpha, m}(x, y)$ given by (1.5) and (1.18) in (2.1), we find the representation

$$
{ }_{H} G_{n}^{\nu, \alpha, m}(x, y)=\sum_{k=0}^{n} \frac{n ! H_{k, m, \alpha}(x, y) G_{n-k}^{\nu, m}(x, y)}{k !} .
$$

Some special cases of $(2.2)$ are

$$
{ }_{H} G_{n}^{\nu, 1, m}(x, y)={ }_{H} C_{n}^{\nu, m}(x, y)=\sum_{k=0}^{n} \frac{n ! H_{k}^{m}(x, y) C_{n-k}^{\nu, m}(x, y)}{k !} .
$$

Here ${ }_{H} C_{n}^{\nu, m}(x, y)$ are Hermite-Gegenbaurer polynomials of two variables.

$$
{ }_{H} C_{n}^{1, m}(x, y)={ }_{H} U_{n}^{m}(x, y)=\sum_{k=0}^{n} \frac{n ! H_{k}^{m}(x, y) U_{n-k}^{m}(x, y)}{k !},
$$

where ${ }_{H} U_{n}^{m}(x, y)$ are Hermite-Chebychev polynomials of two variables.

$$
{ }_{H} C_{n}^{1 / 2, m}(x, y)={ }_{H} P_{n}^{m}(x, y)=\sum_{k=0}^{n} \frac{n ! H_{k}^{m}(x, y) P_{n-k}^{m}(x, y)}{k !}
$$

where ${ }_{H} P_{n}^{m}(x, y)$ are Hermite-Legendre polynomials of two variables.

As a special case, let $y=0$ and $\alpha=2$ be chosen in (2.1) so that generalized Humbert-Hermite polynomial ${ }_{H} G_{n}^{\nu, \alpha, m}(x, y)$ of two variables reduces to HumbertHermite polynomial ${ }_{H} G_{n}^{\nu, 2, m}(x, 0)={ }_{H} G_{n}^{\nu, m}(x)$ of one variable. Then (2.1) yields the 
generating function

$$
\left[1-2 x t+t^{m}\right]^{-\nu} e^{2 x t-t^{m}}=\sum_{n=0}^{\infty}{ }_{H} G_{n}^{\nu, m}(x) t^{n} .
$$

Furthermore, the Hermite-Gegenbaurer (or ultraspherical) polynomials ${ }_{H} C_{n}^{\nu, 2}(x)=$ ${ }_{H} C_{n}^{\nu}(x)$ of one variable, for nonnegative integer $\nu$ are given by

$$
e^{2 x t-t^{2}}\left(1-2 x t+t^{2}\right)^{-\nu}=\sum_{n=0}^{\infty}{ }_{H} C_{n}^{\nu}(x) \frac{t^{n}}{n !} .
$$

Letting $\nu=1 / 2$ and $\nu=1$ in $(2.4)$ gives

$$
e^{2 x t-t^{2}}\left(1-2 x t+t^{2}\right)^{-1 / 2}=\sum_{n=0}^{\infty}{ }_{H} P_{n}(x) \frac{t^{n}}{n !},
$$

where ${ }_{H} P_{n}(x)$ are Hermite-Legendre polynomials and

$$
e^{2 x t-t^{2}}\left(1-2 x t+t^{2}\right)^{-1}=\sum_{n=0}^{\infty}{ }_{H} U_{n}(x) \frac{t^{n}}{n !}
$$

where ${ }_{H} U_{n}(x)$ are Hermite-Chebyshev polynomials.

\section{On expansions of Hermite-Chebyshev and Hermite-Gegenbaurer poly- nomials}

In this section, we prove several theorems on the expansions of Hermite-Gegenbaurer and Hermite-Chebyshev polynomials of two variables. We will start with $(2.1),(2.3)$ and a special case of $(2.1)$ for $\nu=1$,

$$
\left[1-2(x+y) t+t^{m}(2 x y+1)\right]^{-1} e^{\alpha(x+y) t-(x y+1) t^{m}}=\sum_{n=0}^{\infty}{ }_{H} U_{n}^{\alpha, m}(x, y) \frac{t^{n}}{n !},
$$

which will be used in obtaining the corollaries of the following theorem.

Theorem 3.1. For $k \in \mathbb{N}$ and $x, y \in \mathbb{C}$

$$
\begin{aligned}
& \sum_{r=0}^{n} \frac{H_{r}^{m}(\alpha k(x+y),-k(x y+1)) G_{n-r}^{\nu k, m}(x, y)}{r !} \\
= & \sum_{n_{1}+n_{2}+\cdots+n_{k}=n} \frac{{ }_{H} G_{n_{1}}^{\nu, \alpha, m}(x, y)_{H} G_{n_{2}}^{\nu, \alpha, m}(x, y) \cdots_{H} G_{n_{k}}^{\nu, \alpha, m}(x, y)}{n_{1} ! n_{2} ! \cdots n_{k} !} .
\end{aligned}
$$

Proof. The definition of ${ }_{H} G_{n}^{\nu, \alpha, m}(x, y)$ given in (2.1) can be written as

$$
\begin{gathered}
{\left[\left[1-2(x+y) t+t^{m}(2 x y+1)\right]^{-\nu} e^{\alpha(x+y) t-(x y+1) t^{m}}\right]^{k}} \\
=\left[1-2(x+y) t+t^{m}(2 x y+1)\right]^{-\nu k} e^{\alpha k(x+y) t-k(x y+1) t^{m}}=\left[\sum_{n=0}^{\infty}{ }_{H} G_{n}^{\nu, \alpha, m}(x, y) \frac{t^{n}}{n !}\right]^{k} .
\end{gathered}
$$

Using (1.4), we can write

$$
e^{\alpha k(x+y) t-k(x y+1) t^{m}}=\sum_{r=0}^{\infty} H_{r}^{m}(\alpha k(x+y),-k(x y+1)) \frac{t^{r}}{r !} .
$$


Thus it follows that the above result is essentially equivalent to

$$
\begin{gathered}
\sum_{n=0}^{\infty} G_{n}^{\nu k, m}(x, y) t^{n} \sum_{r=0}^{\infty} H_{r}^{m}(\alpha k(x+y),-k(x y+1)) \frac{t^{r}}{r !} \\
=\sum_{n=0}^{\infty} \sum_{n_{1}+n_{2}+\cdots+n_{k}=n} \frac{{ }_{H} G_{n_{1}}^{\nu, \alpha, m}(x, y)_{H} G_{n_{2}}^{\nu, \alpha, m}(x, y) \cdots_{H} G_{n_{k}}^{\nu, \alpha, m}(x, y)}{n_{1} ! n_{2} ! \cdots n_{k} !} t^{n} .
\end{gathered}
$$

An application of manipulation of series yields

$$
\begin{gathered}
\sum_{n=0}^{\infty} \sum_{r=0}^{n} \frac{H_{r}^{m}(\alpha k(x+y),-k(x y+1)) G_{n-r}^{\nu k, m}(x, y)}{r !} t^{n} \\
=\sum_{n=0}^{\infty} \sum_{n_{1}+n_{2}+\cdots+n_{k}=n} \frac{{ }_{H} G_{n_{1}}^{\nu, \alpha, m}(x, y)_{H} G_{n_{2}}^{\nu, \alpha, m}(x, y) \cdots_{H} G_{n_{k}}^{\nu, \alpha, m}(x, y)}{n_{1} ! n_{2} ! \cdots n_{k} !} t^{n} .
\end{gathered}
$$

Now equating coefficients of $t^{n}$ on both sides of the resulting equation will give the required result.

Remark 3.1. Setting $\nu=1$ in Theorem 3.1, the result reduces to

Corollary 3.1. For $k \in \mathbb{N}$ and $x, y \in \mathbb{C}$

$$
\begin{aligned}
& \sum_{r=0}^{n} \frac{H_{r}^{m}(\alpha k(x+y),-k(x y+1)) C_{n-r}^{k, m}(x, y)}{r !} \\
= & \sum_{n_{1}+n_{2}+\cdots+n_{k}=n} \frac{{ }_{H} U_{n_{1}}^{\alpha, m}(x, y)_{H} U_{n_{2}}^{\alpha, m}(x, y) \cdots{ }_{H} U_{n_{k}}^{\alpha, m}(x, y)}{n_{1} ! n_{2} ! \cdots n_{k} !} .
\end{aligned}
$$

Remark 3.2. Setting $\nu=0$ in Theorem 3.1, the result reduces to

Corollary 3.2. For $k \in \mathbb{N}$ and $x, y \in \mathbb{C}$

$$
\begin{gathered}
\frac{H_{n}^{m}(\alpha k(x+y),-k(x y+1))}{n !} \\
=\sum_{n_{1}+n_{2}+\cdots+n_{k}=n} \frac{H_{n_{1}}^{\alpha, m}(x, y) H_{n_{2}}^{\alpha, m}(x, y) \cdots H_{n_{k}}^{\alpha, m}(x, y)}{n_{1} ! n_{2} ! \cdots n_{k} !} .
\end{gathered}
$$

Remark 3.3. Setting $\alpha=m=2, \nu, y=0$ in Theorem 3.1, the result reduces to known result of Batahan and Shehata [2,p.50.,Eq.(2.1)].

Corollary 3.3. For $k \in \mathbb{N}$ and $x \in \mathbb{C}$

$$
\sum_{r=0}^{\left[\frac{n}{2}\right]} \frac{(-k)^{r}(2 k x)^{n-2 r}}{(n-2 r) r !}=\sum_{n_{1}+n_{2}+\cdots+n_{k}=n} \frac{H_{n_{1}}(x) H_{n_{2}}(x) \cdots H_{n_{k}}(x)}{n_{1} ! n_{2} ! \cdots n_{k} !} .
$$

Theorem 3.2. For $k \in \mathbb{N}$ and $X, Y \in \mathbb{C}$

$$
\begin{gathered}
\sum_{r=0}^{n} \frac{H_{r}^{m}(\alpha k(X+Y),-k(X Y+1)) G_{n-r}^{\nu k, m}(X, Y)}{r !} \\
=\sum_{n_{1}+n_{2}+\cdots+n_{k}=n} \frac{{ } G_{n_{1}}^{\nu, \alpha, m}(X, Y)_{H} G_{n_{2}}^{\nu, \alpha, m}(X, Y){ }^{\prime}{ }_{H} G_{n_{k}}^{\nu, \alpha, m}(X, Y)}{n_{1} ! n_{2} ! \cdots n_{k} !}
\end{gathered}
$$

where $X=\sum_{i=0}^{k} x_{i}$ and $Y=\sum_{j=0}^{k} y_{j}$. 
Proof. The definition of ${ }_{H} G_{n}^{\nu, \alpha, m}(x, y)$ can be written as

$$
\begin{aligned}
& {\left[\left[1-2(X+Y) t+t^{m}(2 X Y+1)\right]^{-\nu} e^{\alpha(X+Y) t-(X Y+1) t^{m}}\right]^{k} } \\
= & {\left[1-2(X+Y) t+t^{m}(2 X Y+1)\right]^{-\nu k} e^{\alpha k(X+Y) t-k(X Y+1) t^{m}} } \\
= & {\left[\sum_{n=0}^{\infty}{ }_{H} G_{n}^{\nu, \alpha, m}\left(x_{1}+x_{2}+\cdots+x_{k}, y_{1}+y_{2}+\cdots+y_{k}\right) \frac{t^{n}}{n !}\right]^{k} . }
\end{aligned}
$$

Using (1.4), we can write

$$
e^{\alpha k(X+Y) t-k(X Y+1) t^{m}}=\sum_{r=0}^{\infty} H_{n}^{m}(\alpha k(X+Y),-k(X Y+1)) \frac{t^{r}}{r !} .
$$

Thus it follows that the above result is essentially equivalent to

$$
\begin{gathered}
\sum_{n=0}^{\infty} G_{n}^{\nu k, m}(X, Y) t^{n} \sum_{r=0}^{\infty} H_{n}^{m}(\alpha k(X+Y),-k(X Y+1)) \frac{t^{r}}{r !} \\
=\sum_{n=0}^{\infty} \sum_{n_{1}+n_{2}+\cdots+n_{k}=n} \frac{{ }_{H} G_{n_{1}}^{\nu, \alpha, m}(X, Y)_{H} G_{n_{2}}^{\nu, \alpha, m}(X, Y) \cdots{ }_{H} G_{n_{k}}^{\nu, \alpha, m}(X, Y)}{n_{1} ! n_{2} ! \cdots n_{k} !} t^{n} .
\end{gathered}
$$

An application of manipulation of series yields

$$
\begin{gathered}
\sum_{n=0}^{\infty} \sum_{r=0}^{n} \frac{H_{r}^{m}(\alpha k(X+Y),-k(X Y+1)) G_{n-r}^{\nu k, m}(X, Y)}{r !} t^{n} \\
=\sum_{n=0}^{\infty} \sum_{n_{1}+n_{2}+\cdots+n_{k}=n} \frac{{ }_{H} G_{n_{1}}^{\nu, \alpha, m}(X, Y)_{H} G_{n_{2}}^{\nu, \alpha, m}\left(X, Y \cdots{ }_{H} G_{n_{k}}^{\nu, \alpha, m}(X, Y)\right.}{n_{1} ! n_{2} ! \cdots n_{k} !} t^{n} .
\end{gathered}
$$

Now equating coefficients of $t$ on both sides of the resulting equation will give the required result.

Remark 3.4. Setting $\nu=1$ in Theorem 3.2, the result reduces to

Corollary 3.4. For $k \in \mathbb{N}$ and $x, y \in \mathbb{C}$, we have

$$
\begin{aligned}
& \sum_{r=0}^{n} \frac{H_{r}^{m}(\alpha k(X+Y),-k(X Y+1)) C_{n-r}^{k, m}(X, Y)}{r !} \\
= & \sum_{n_{1}+n_{2}+\cdots+n_{k}=n} \frac{{ }_{H} U_{n_{1}}^{\alpha, m}(X, Y)_{H} U_{n_{2}}^{\alpha, m}(X, Y) \cdots_{H} U_{n_{k}}^{\alpha, m}(X, Y)}{n_{1} ! n_{2} ! \cdots n_{k} !} .
\end{aligned}
$$

Remark 3.5. Setting $\nu=0$ in Theorem 3.2, the result reduces to

Corollary 3.5. For $k \in \mathbb{N}$ and $X, Y \in \mathbb{C}$, we have

$$
\begin{gathered}
\frac{H_{n}^{m}(\alpha k(X+Y),-k(X Y+1))}{n !} \\
=\sum_{n_{1}+n_{2}+\cdots+n_{k}=n} \frac{H_{n_{1}}^{\alpha, m}(X, Y) H_{n_{2}}^{\alpha, m}(X, Y) \cdots H_{n_{k}}^{\alpha, m}(X, Y)}{n_{1} ! n_{2} ! \cdots n_{k} !} .
\end{gathered}
$$

Remark 3.6. Setting $\alpha=m=2, \nu=0, x_{2}=\cdots x_{k}=0, y_{1}=\cdots y_{k}=0$ and replacing $x_{1}$ by $x$ in Theorem 3.2, the result reduces to known result of Batahan and Shehata [2,p.51.,Eq.(2.4)]. 
Corollary 3.6. For $k \in \mathbb{N}$ and $x \in \mathbb{C}$, we have

$$
\sum_{r=0}^{\left[\frac{n}{2}\right]} \frac{(-k)^{r}(2 k x)^{n-2 r}}{(n-2 r) r !}=\sum_{n_{1}+n_{2}+\cdots+n_{k}=n} \frac{H_{n_{1}}(x) H_{n_{2}}(x) \cdots H_{n_{k}}(x)}{n_{1} ! n_{2} ! \cdots n_{k} !} .
$$

Theorem 3.3. For $k \in \mathbb{N}$ and $x, y \in \mathbb{C}$, we have

$$
\begin{aligned}
& \sum_{s=0}^{\left[\frac{n}{m}\right]} \frac{(-1)^{s}(\nu k)_{n-(m-1) s}(2 x+2 y)^{n-m s}(2 x y+1)^{s}}{s !(n-m s) !} \\
= & \sum_{n_{1}+n_{2}+\cdots+n_{k}=n} G_{n_{1}}^{\nu, m}(x, y) G_{n_{2}}^{\nu, m}(x, y) \cdots G_{n_{k}}^{\nu, m}(x, y) .
\end{aligned}
$$

Proof. Using the power series of $\left[1-2(x+y) t+t^{m}(2 x y+1)\right]^{-k}$ and making the necessary series arrangements gives

$$
\left[1-2(x+y) t+t^{m}(2 x y+1)\right]^{-\nu k}=\sum_{n=0}^{\infty} \sum_{s=0}^{\left[\frac{n}{m}\right]} \frac{(-1)^{s}(\nu k)_{n-(m-1) s}(2 x+2 y)^{n-m s}(2 x y+1)^{s}}{s !(n-m s) !} t^{n} .
$$

In addition to this, we can write

$$
\begin{gathered}
{\left[1-2(x+y) t+t^{m}(2 x y+1)\right]^{-k}=\left[\left[1-2(x+y) t+t^{m}(2 x y+1)\right]^{-\nu}\right]^{k}=\left[\sum_{n=0}^{\infty} G_{n}^{\nu, m}(x, y) t^{n}\right]^{k}} \\
=\sum_{n=0}^{\infty} \sum_{n_{1}+n_{2}+\cdots+n_{k}=n} G_{n_{1}}^{\nu, m}(x, y) G_{n_{2}}^{\nu, m}(x, y) \cdots G_{n_{k}}^{\nu, m}(x, y) t^{n}
\end{gathered}
$$

Now equating coefficients of $t$ on both sides of the resulting equation will give the required result.

Remark 3.7. For $\nu=1$ in Theorem 3.3, the result reduces to

Corollary 3.7. For $k \in \mathbb{N}$ and $x, y \in \mathbb{C}$, we have

$$
\begin{aligned}
& \sum_{s=0}^{\left[\frac{n}{m}\right]} \frac{(-1)^{s}(k)_{n-(m-1) s}(2 x+2 y)^{n-m s}(2 x y+1)^{s}}{s !(n-m s) !} \\
& =\sum_{n_{1}+n_{2}+\cdots+n_{k}=n} U_{n_{1}}^{m}(x, y) U_{n_{2}}^{m}(x, y) \cdots U_{n_{k}}^{m}(x, y) .
\end{aligned}
$$

Acknowledgement: The first author M. A. Pathan would like to thank the Department of Science and Technology, Government of India, for the financial assistance for this work under project number SR/S4/MS:794/12.

\section{References}

[1] Andrews, L. C, Special functions for engineers and mathematicians, Macmillan Co. New York, 1985.

[2] Batahan, R. S and Shehata, A, Hermite-Chebyshev polynomials with their generalization form, J. Math. Sci. Adv. Appl., 29 (2014), 47-59.

[3] Bell, E. T, Exponential polynomials, Ann. of Math., 35(1934), 258-277.

[4] Dave, C. K, Another generalization of Gegenbauer polynomials, J. Indian Acad. Math., 1, 2 (1978), 42-45. 
[5] Dattoli, G, Chiccoli, C, Lorenzutta, S, Maimo, G and Torre, A, Generalized Bessel functions and generalized Hermite polynomials, J. Math. Anal. Appl., 178(1993), 509-516.

[6] Dattoli, G, Maimo, G, Torre, A and Cesarano, C, Generalized Hermite polynomials and super-Gaussian forms, J. Math. Anal. Appl., 233(1996), 597-609.

[7] Dattoli, G, Lorenzutta, S and Cesarano, C, Finite sums and generalized forms of Bernoulli polynomials, Rendiconti di Mathematica, 19(1999), 385-391.

[8] Dattoli, G, Torre, A and Lorenzutta, S, Operational identities and properties of ordinary and generalized special functions, J. Math. Anal. Appl., 236(1999), 399-414.

[9] Djordjević , G. B, A generalization of Gegenbauer polynomial with two variables, (To appear in Indian J. Pure Appl. Math).

[10] Djordjević, G. B and Milovanović ,G.V, Special Classes of Polynomials, University of Nis, Faculty of Technology, Leskovac, 2014.

[11] Gould, H. W, Inverse series relation and other expansions involving Humbert polynomials, Duke Math. J., 32(1965), 697-711.

[12] Horadam, A, Gegenbauer polynomials revisited, The Fibonacci Quart., 23 (1985), 295-299, 307.

[13] Horadam, A and Pethe, S, Polynomials associated with Gegenbauer polynomials, The Fibonacci Quart., 19(1981), 393-398.

[14] Milovanović , G. V and Djordjević , G. B, On some properties of Humberts polynomials-I, Fibonacci Quart., 25 (1987), 356-360

[15] Milovanović , G. V and Djordjević , G. B, On some properties of Humberts polynomials-II, Facta Universitatis (Nis) Math. Inform., 6 (1991), 23-30.

[16] Pathan, M. A and Khan, M. A, On polynomials associated with Humberts polynomials, Publ. Inst. Math., (Beograd), 62(76)(1997), 53-62.

[17] Pathan, M. A and Khan, N. U, On unified presentation of a class of generalized Humberts polynomials of two variables, ROMAI J., 11(2)(2015), 185-199.

[18] Shrestha, N. B, Polynomial associated with Legendre polynomials, Nepali Math. Sci. Rep. Triv. Univ., 2:1 (1977).

[19] Sinha, S. K, On a polynomial associated with Gegenbauer polynomials, Proc. Nat. Acad. Sci., India, 59 (A):III (1989), 439-455. 